\title{
Reações Adversas Imunomediadas em pacientes tratados com Inibidores de Checkpoints Imunológicos em um Hospital filantrópico de Salvador
}

\author{
Immunomedied Adverse Reactions in patients treated with Immunological Checkpoints Inhibitors \\ in a filantropic Hospital in Salvador \\ Reacciones Adversas Inmunomediadas en pacientes tratados con Inhibidores de Puntos de Control \\ Inmunológicos en un Hospital Filantropico de Salvador
}

Recebido: 12/02/2021 | Revisado: 20/02/2021 | Aceito: 23/02/2021 | Publicado: 28/02/2021

\author{
Juliana Pereira dos Santos \\ ORCID: https://orcid.org/0000-0002-5046-743X \\ Universidade do Estado da Bahia, Brasil \\ E-mail: jupereira630@gmail.com \\ Martamaria de Souza Ferraz Ribeiro \\ ORCID: https://orcid.org/0000-0001-8300-6229 \\ Hospital Santa Izabel, Brasil \\ E-mail: martasfribeiro@gmail.com \\ Maria Teresita Bendicho \\ ORCID: https://orcid.org/0000-0001-8234-1199 \\ Universidade do Estado da Bahia, Brasil \\ E-mail: mtbendicho@gmail.com \\ Geraldo Bezerra da Silva Júnior \\ ORCID: https://orcid.org/0000-0002-8971-0994 \\ Universidade de Fortaleza, Brasil \\ E-mail: geraldobezerrajr@unifor.br \\ Rosa Malena Fagundes Xavier \\ ORCID: https://orcid.org/0000-0002-3203-8949 \\ Universidade do Estado da Bahia, Brasil \\ E-mail: rxavier@uneb.br
}

\begin{abstract}
Resumo
Introdução: Os Inibidores de Checkpoints Imunológicos (ICI) bloqueiam os efeitos inibitórios em receptores como o CTLA-4, PD-1 e PD-L1 e reestabelecem a imunidade antitumoral. Dessa maneira, estão associados a Reações adversas Imunomediadas (RAim) gastrintestinais, dermatológicas, hepáticas e endócrinas. Objetivo: Analisar o perfil das reações adversas imunomediadas em pacientes oncológicos tratados com ICI em um hospital de Salvador/Ba. Método: Trata-se de um estudo observacional, transversal, retrospectivo. Foram avaliadas todas as notificações de reações adversas associadas ao tratamento oncológico com os ICI, documentadas no período de janeiro de 2015 a maio de 2020. A pesquisa foi aprovada pelo Comitê de Ética em Pesquisa, conforme o $\mathrm{n}^{\circ}$ do parecer 4.074.757/2020. Resultados: Durante o período do estudo, 27 pacientes fizeram uso de ICI. Quanto as RAim, 17 desenvolveram RAim. Das RAim mais frequentes, $62 \%$ envolveram o Nivolumabe e $21 \%$ o Pembrolizumabe. $26 \%$ das RAim foram reações gastrintestinais, seguido de 19\% reações diversas (como sangramento vaginal e neuropatia); $53 \%$ das RAim foram classificadas como reações moderadas. Dentre as medidas terapêuticas adotadas para resolução das RAim, $73 \%$ dos ICI foram descontinuados e substituídos por outros quimioterápicos; $53 \%$ dos pacientes utilizaram terapias de suporte para manejo de sintomas provocados pelas Raim. Quanto ao desfecho clínico, 53\% pacientes se recuperaram das RAim, $24 \%$ se recuperaram com sequela e $23 \%$ foram a óbito. Conclusão: A introdução de terapêutica dirigida, acompanhamento farmacoterapêutico adequado e o monitoramento pela farmacovigilância favorecerão a identificação de reações adversas imunomediadas, e o uso seguro dos ICI.
\end{abstract}

Palavras-chave: Câncer; Imunoterapia; Imunomoduladores; Reações adversas.

\begin{abstract}
Introduction: Immune Checkpoint Inhibitors (ICI) block the inhibitory effects on receptors such as CTLA-4, PD-1 and PD-L1 and reestablish anti-tumor immunity. Thus, they are associated with gastrointestinal, dermatological, hepatic and endocrine Immune-Mediated Adverse Reactions (RAim). Objective: To analyze the profile of immune-mediated adverse reactions in cancer patients treated with immunological checkpoint inhibitors at a hospital in Salvador / Ba. Method: This is an observational, cross-sectional, retrospective study. All reports of adverse reactions associated with cancer treatment with ICIs, documented between January 2015 and May 2020, were evaluated. The research was approved by the Research Ethics Committee, according to the opinion number 4.074 .757 / 2020. Results: During the
\end{abstract}


study period, 27 patients used ICI. As for the RAim, 17 developed RAim. Of the most frequent RAim, $62 \%$ involved Nivolumab and $21 \%$ Pembrolizumab. $26 \%$ of RAim were gastrointestinal reactions, followed by $19 \%$ various reactions (such as vaginal bleeding and neuropathy); 53\% of RAim were classified as moderate reactions. Among the therapeutic measures adopted to resolve RAIM, 73\% of the ICI were discontinued and replaced by other chemotherapeutic agents; $53 \%$ of patients used supportive therapies to manage symptoms caused by Raim. As for the clinical outcome, $53 \%$ patients recovered from RAim, $24 \%$ recovered with sequelae and $23 \%$ died. Conclusion: The introduction of targeted therapy, adequate pharmacotherapeutic follow-up and monitoring by pharmacovigilance will favor the identification of immune-mediated adverse reactions, and the safe and appropriate use of ICI.

Keywords: Cancer; Immunotherapy; Immunomodulators; Adverse reactions.

\section{Resumen}

Introducción: Los inhibidores de puntos de control inmunológico (ICI) bloquean los efectos inhibidores sobre receptores como CTLA-4, PD-1 y PD-L1 y restablecen la inmunidad antitumoral. Por lo tanto, se asocian con reacciones adversas mediadas por la inmunidad (RAim) gastrointestinales, dermatológicas, hepáticas y endocrinas. Objetivo: Analizar el perfil de reacciones adversas inmunomediadas en pacientes oncológicos tratados con inhibidores de puntos de control inmunológicos en un hospital de Salvador / Ba. Método: Se trata de un estudio observacional, transversal y retrospectivo. Se evaluaron todas las notificaciones de reacciones adversas asociadas al tratamiento del cáncer con ICI, documentadas entre enero de 2015 y mayo de 2020. La investigación fue aprobada por el Comité de Ética en Investigación, de acuerdo al dictamen número 4.074.757 / 2020. Resultados: Durante el período de estudio, 27 pacientes utilizaron ICI. En cuanto a RAim, 17 desarrollaron RAim. De las RAim más frecuentes, el 62\% involucró a Nivolumab y el $21 \%$ a Pembrolizumab. El $26 \%$ de los RAim fueron reacciones gastrointestinales, seguidas de un $19 \%$ de reacciones diversas (como hemorragia vaginal y neuropatía); El 53\% de los RAim se clasificaron como reacciones moderadas. Entre las medidas terapéuticas adoptadas para resolver la RAIM, el 73\% de las ICI fueron discontinuadas y reemplazadas por otros agentes quimioterapéuticos; El 53\% de los pacientes utilizaron terapias de apoyo para controlar los síntomas causados por Raim. En cuanto al resultado clínico, el 53\% de los pacientes se recuperaron de RAim, el 24\% se recuperó con secuelas y el 23\% falleció. Conclusión: La introducción de la terapia dirigida, el seguimiento farmacoterapéutico adecuado y la monitorización por farmacovigilancia favorecerán la identificación de reacciones adversas inmunomediadas y el uso seguro y apropiado de ICI.

Palabras clave: Cáncer; Inmunoterapia; Inmunomoduladores; Reacciones adversas.

\section{Introdução}

O câncer é a segunda maior causa de morte no mundo, tendo levado a óbito cerca de 9,6 milhões de pessoas só no ano de 2018. Um dos maiores desafios para conter o avanço da doença consiste no desenvolvimento de formas mais eficazes de tratamento. Nos últimos anos, novas terapias são dirigidas visando a identificação e reversão dos mecanismos de escape do tumor, com o objetivo de regularizar a capacidade antitumoral do sistema imunológico. Tratamentos direcionados para a modulação da resposta imunológica ao microambiente do tumor apresentam um resultado eficiente e menos efeitos adversos, em comparação a terapias convencionais que estimulam de forma sistêmica os mecanismos de resposta imune (Teixeira; Silva; Menão \& Oliveira, 2019).

Os inibidores dos checkpoints imunológicos revolucionaram o tratamento de muitos tipos diferentes de câncer. São receptores responsáveis pela redução da atividade imunitária necessária para evitar autoimunidade e possibilitar a tolerância imunológica. As proteínas do ponto de verificação imune associados linfócitos T citotóxicos 4 (CTLA-4) e à proteína 1 de morte celular programada (PD-1) são receptores expressos na superfície de células $\mathrm{T}$ citotóxicas que interagem com seus ligantes CD80 / diferencial de agrupamento 86 (CD86) e ligante de morte celular programada 1 (PD-L1) em célula apresentadora de antígeno (APCs). Essas vias podem ser cooptadas para ajudar as células cancerígenas a evitar a morte mediada por células T citotóxicas. O bloqueio dos efeitos inibitórios do CTLA-4, PD-1 e PD-L1, reestabelece a imunidade antitumoral mediada por células $\mathrm{T}$, permitindo a estimulação antitumoral do sistema imunológico e aumentando as respostas contra células neoplásicas (Brahmer, et al. 2018).

Foram aprovados no Brasil anticorpos monoclonais voltados a correceptores inibitórios envolvidos na modulação da sinapse imune para uso clínico, incluindo o agente anti-CTLA-4, ipilimumabe, que foi o primeiro agente aprovado para uso em casos de melanoma avançado. E os agentes anti-PD-1, pembrolizumabe e o nivolumabe foram aprovados para melanoma, 
câncer de pulmão metastático de não pequenas células, câncer de cabeça e pescoço, carcinoma urotelial, adenocarcinoma gástrico e tumores sólidos com deficiência de reparo e incompatibilidade para linfoma de Hodgkin clássico (Barclay, Creswell, \& León, 2018).

Ademais, o nivolumabe foi aprovado para uso em carcinoma hepatocelular e pacientes com carcinoma de células renais. Para pacientes com melanoma avançado foi aprovado pela Food and Drug Administration (FDA) a combinação de ipilimumabe e nivolumabe. Mais recentemente, os anticorpos PD-L1 atezolizumabe (aprovado para uso em câncer urotelial, câncer de pulmão metastático de não pequenas células e câncer de mama), durvalumabe (aprovado para uso em câncer urotelial) e avelumabe (aprovado para uso em carcinoma de células de Merkel e carcinoma urotelial) também foram desenvolvidos para bloquear a via PD-1. Assim, tanto as indicações de uso continuam a se expandir, como o desenvolvimento de novos agentes e combinações de vários inibidores dos checkpoints imunológicos continuam a serem avaliados para múltiplas indicações (Wainstein, et al. 2017; Regalla, Williams, \& kumar, 2018).

Contudo, esta estimulação excessiva do sistema imunológico pode provocar o surgimento de efeitos adversos imunomediados. Tais reações resultam da ativação do sistema imunológico e frequentemente apresentam sintomas generalizados, incluindo fadiga ou febre e, em alguns pacientes, podem causar danos específicos a órgãos. Os alvos mais frequentes da toxicidade dos inibidores de checkpoints são a pele, intestino, sistema endócrino, pulmão e musculoesquelético, enquanto que as reações adversas cardiovasculares, hematológicas, renais, neurológicas e oftalmológicas ocorrem com muito menos frequência (Abdel, 2019).

Embora a maioria das reações adversas imunomediadas sejam de grau leve ou moderado, por vezes alguns pacientes desenvolvem reações graves ou fatais, como no caso de colite grave, pneumonite, encefalite, necrólise epidérmica tóxica, miocardite e cetoacidose diabética, gerando uma média de mortalidade entre $2 \%$. As medidas terapêuticas normalmente envolvem o uso de esteroides e outras estratégias de imunossupressão, bem como suspensão temporária ou permanente do inibidor de checkpoint (Lleo, Rimassa, \& Colombo, 2019). Assim, este estudo visa caracterizar o perfil das reações imunomediadas em pacientes com câncer tratados com inibidores dos checkpoints imunológicos em um hospital filantrópico de Salvador, Bahia, Brasil.

\section{Metodologia}

Realizou-se um estudo observacional, retrospectivo, quantitativo, descritivo, com delineamento transversal, conforme descrito por Pereira et al., (2018), através da análise dos prontuários e notificações de reações adversas imunomediadas de pacientes oncológicos que fizeram uso de terapia com inibidores dos checkpoints imunológicos durante o período de janeiro de 2015 a maio de 2020, no setor de Farmácia localizado no Instituto Baiano de Câncer do Hospital Santa Izabel. O presente estudo foi aprovado pelo Comitê de Ética e Pesquisa da Universidade do Estado da Bahia, com o número do parecer: 4.074.757/2020.

As variáveis analisadas foram os aspectos sociodemográficas, clínicos e farmacológicas, incluindo faixa etária, gênero, escolaridade, origem, tipo de câncer, estadiamento do câncer, tratamentos realizados, morbidades associadas, alergias; os principais aspectos clínicos relacionados às reações adversas imunomediadas; as medidas terapêuticas adotadas para reverter as reações adversas; e os desfechos clínicos observados para cada caso.

Foram incluídas apenas as notificações de reações adversas imunomediadas que foram tratadas pelo setor de farmacovigilância do hospital, em pacientes com idade igual ou superior a dezoito anos e que foram enviadas à Agência Nacional de Vigilância Sanitária (ANVISA), no período de janeiro de 2015 a maio de 2020. Foram excluídas as reações ocorridas fora do período proposto no estudo, pacientes com idade inferior a dezoito anos e as que não foram devidamente notificadas. 
A análise estatística foi realizada através das variáveis analisadas e correlacionadas. Para o processamento e análise dos dados foi utilizado o Programa Software for Statitics and Data Science - STATA® 16.0. Sendo realizada a distribuição de frequência absoluta e relativa das variáveis estudadas, organizadas em tabelas e dados. Para associações entre as variáveis foram aplicados os testes Qui-quadrado e o teste exato de Fisher e nos casos aplicáveis, a Razão de Chances (Odds Ratio), com intervalo de confiabilidade de $95 \%$, sendo considerado estatisticamente significante o valor p associado menor ou igual a 0,05 $(\mathrm{p} \leq 0,05)$.

\section{Resultados}

No período compreendido entre janeiro de 2015 a maio de 2020, 28 pacientes fizeram uso de inibidores de checkpoints imunológicos e 17 (65\%) desenvolveram reações adversas imunomediadas. Destes, 1 paciente foi excluído da pesquisa por insuficiência de informações, sendo incluídos apenas 27 pacientes.

De acordo com as características sociodemográficas, presentes na Tabela 1, 52\% dos pacientes oncológicos em tratamento com os ICI eram homens e $48 \%$ eram mulheres, encontrando-se 59\% na faixa etária de $60-89$ anos. $71 \%$ dos pacientes eram pardos, $59 \%$ eram casados, $63 \%$ tendo cursado até o nível médio. Em relação à localização do domicílio, $89 \%$ dos pacientes residiam em Salvador e 11\% dos pacientes residiam em outras cidades da Bahia, conforme Tabela 1. 
Tabela 1. Características sociodemográficas dos pacientes oncológicos tratados com inibidores do checkpoint imunológico que apresentaram reações adversas imunomediadas, em hospital filantrópico de Salvador, Bahia, Brasil.

\begin{tabular}{lcc} 
Sexo & $\mathbf{N}^{\mathbf{0}}$ & $\%$ \\
\hline Feminino & 13 & 48 \\
Masculino & 14 & 52 \\
Faixa Etária & & 15 \\
$\mathbf{1 8 - 3 9}$ & 4 & 26 \\
$\mathbf{4 0 - 5 9}$ & 7 & 59 \\
$\mathbf{6 0 - 8 9}$ & 16 &
\end{tabular}

\section{Escolaridade}

Não informado

Nível fundamental

Nível médio

Nível superior

\section{Estado Civil}

Solteiro (a)

Casado (a)

Divorciado (a)

Viúvo (a)

Viúvo (a)

\section{Etnia}

\section{Preto}

Branco

Pardo

\section{Origem}

Salvador

Outras cidades

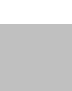

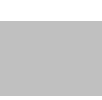

4

3

17

3

\section{4}

$\sqrt{10}$

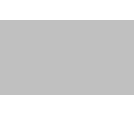

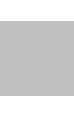

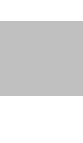

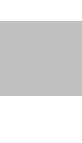

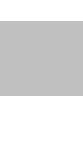

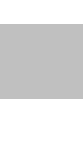

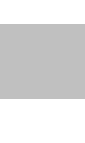

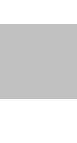

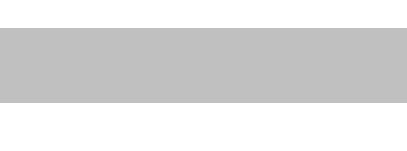

1

$\begin{array}{ll}1 & 23 \\ 12 & 71\end{array}$

12

\section{$\%$


Tabela 2. Características clínicas dos pacientes oncológicos tratados com inibidores do checkpoint imunológico que apresentaram reações adversas imunomediadas, em hospital filantrópico de Salvador, Bahia, Brasil.

\begin{tabular}{|c|c|c|}
\hline T ípos de câncer & $\mathbf{N}^{\circ}$ & $\%$ \\
\hline Câncer de pulmão & 11 & 41 \\
\hline Melanoma & 7 & 29 \\
\hline Câncer de bexiga & 2 & 7 \\
\hline Carcinoma renal de células claras & 2 & 7 \\
\hline Câncer de mama & 1 & 4 \\
\hline Câncer de orofaringe & 1 & 4 \\
\hline L infoma de Hodgkin & 1 & 4 \\
\hline Adenocarcinoma gástrico & 1 & 4 \\
\hline \multicolumn{3}{|l|}{ E stadiamento anatomop atológico } \\
\hline II & 1 & 4 \\
\hline III & 4 & 15 \\
\hline IV & 22 & 81 \\
\hline \multicolumn{3}{|l|}{ A lergias } \\
\hline Sim & 7 & 26 \\
\hline Não & 20 & 74 \\
\hline \multicolumn{3}{|c|}{ Inibidores de checkpoints usados no tratamento oncológico } \\
\hline Pembrolizumabe & 12 & 44 \\
\hline Nirolumabe & 11 & 41 \\
\hline Ipilimumabe & 3 & 11 \\
\hline A tezolizumabe & 1 & 4 \\
\hline \multicolumn{3}{|l|}{ Comorbidades } \\
\hline D iabetes mellitus 2 & 11 & 23 \\
\hline Hị ertensão arterial sistêmica & 9 & 19 \\
\hline T rombose & 6 & 12 \\
\hline Dislipidemia & 4 & 8 \\
\hline Doença renal & 3 & 6 \\
\hline Fibrilação atriale rônica & 2 & 4 \\
\hline Dor oncológica & 2 & 4 \\
\hline Câncer & 2 & 4 \\
\hline Doença arterial coronariana & 2 & 4 \\
\hline Hip otir eoid ismo & 2 & 4 \\
\hline Outras (DPOC; DRGE ; d epressão) & 3 & 6 \\
\hline Sem comorbidades & 3 & 6 \\
\hline
\end{tabular}

Fonte: Autores. 
Foram observadas 26 reações adversas imunomediadas, 43\% das reações foram classificadas como outras manifestações (inefetividade; sangramento vaginal; alteração visual; sonolência; astenia; edema; neutropenia; plaquetopenia; neuropatia periférica; dermatite; prurido; erupção cutânea), 24\% envolveram reações gastrintestinais, $14 \%$ reações endócrinas e metabólicas e $14 \%$ reações pulmonares. Em relação aos inibidores de checkpoints mais associados às RAim, o Nivolumabe ocasionou $62 \%$ das reações e o Pembrolizumabe $21 \%$. A Tabela 3 retrata os principais dados relacionados as RAim associadas aos ICI.

Ainda de acordo com a Tabela 3, entre os quatro ICI, o nivolumabe apresentou $21 \%$ de reações classificadas como outras manifestações e $16 \%$ de reações gastrintestinais. As reações adversas relacionadas ao tratamento predominantes para pembrolizumabe foram às outras manifestações com 16\% e 5\% foram reações pulmonares. Os principais RAim relacionados ao tratamento com ipilimumabe foram gastrintestinais com $5 \%$ e outras manifestações $5 \%$. O atezolizumabe apresentou apenas reações gastrintestinais com $2 \%$.

Tabela 3. Reações adversas imunomediadas causadas por inibidores do checkpoint imunológico usados no tratamento oncológico, em hospital filantrópico de Salvador, Bahia, Brasil.

\begin{tabular}{|c|c|c|c|c|c|}
\hline \multirow{2}{*}{$\begin{array}{l}\text { REAÇÕES ADVERSAS } \\
\text { IMUNOMEDIADAS }\end{array}$} & \multicolumn{4}{|c|}{ INIBIDORES DO CHECKPOINT IMUNOLÓGICO } & \multirow[b]{2}{*}{ Total } \\
\hline & Nivolumabe & Pembrolizumabe & Ipilimumabe & Atezolizumabe & \\
\hline Gastrintestinais & $7(16 \%)$ & & $2(5 \%)$ & $1(2 \%)$ & $10(24 \%)$ \\
\hline Hepatotoxicidade & $1(2 \%)$ & & $1(2 \%)$ & & $2(5 \%)$ \\
\hline Pulmonares & $4(9 \%)$ & $2(5 \%)$ & & & $6(14 \%)$ \\
\hline Endócrinos & $5(12 \%)$ & & $1(2 \%)$ & & $6(14 \%)$ \\
\hline Outras manifestações & $9(21 \%)$ & $7(16 \%)$ & $2(5 \%)$ & & $18(43 \%)$ \\
\hline Total & $26(62 \%)$ & $9(21 \%)$ & $6(14 \%)$ & $1(2 \%)$ & 42 \\
\hline
\end{tabular}

Fonte: Autores.

De acordo com a Figura 1, 53\% foram classificadas como reações moderadas e 47\% como reações graves. Dentre as medidas terapêuticas adotadas para resolução das RAim, 73\% dos inibidores de checkpoints foram descontinuados e substituídos por outros quimioterápicos, $11 \%$ dos medicamentos foram suspensos e reintroduzidos e $11 \%$ os medicamentos não foram suspensos. 53\% dos pacientes utilizaram terapias de suporte para manejo de sintomas provocados pelas Raim, como corticoides, antieméticos, antidiarreicos, terapia de reposição hormonal. 
Figura 1. Gravidade das reações imunomediadas após tratamento inibidores do checkpoint imunológico e medidas terapêuticas adotadas para manejo clínico.

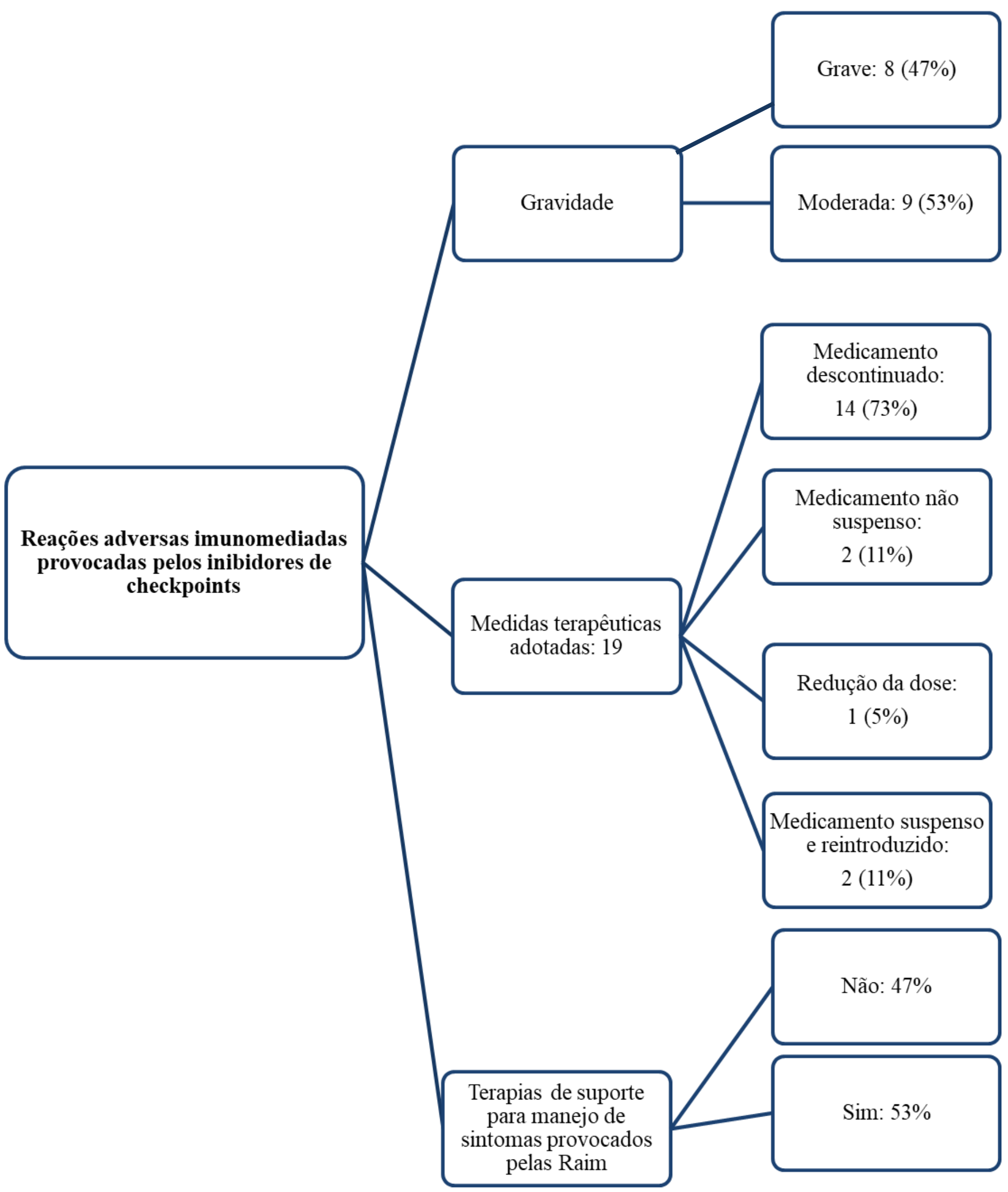

Fonte: Autores. 
Quanto ao desfecho clínico, 53\% pacientes se recuperaram das RAim, 24\% se recuperaram com sequela e 23\% foram a óbito, como se segue no Gráfico 1.

Gráfico 1. Desfecho clínico dos pacientes oncológicos que apresentaram reações imunomediadas após tratamento com inibidores do checkpoint imunológico.

$53 \%$

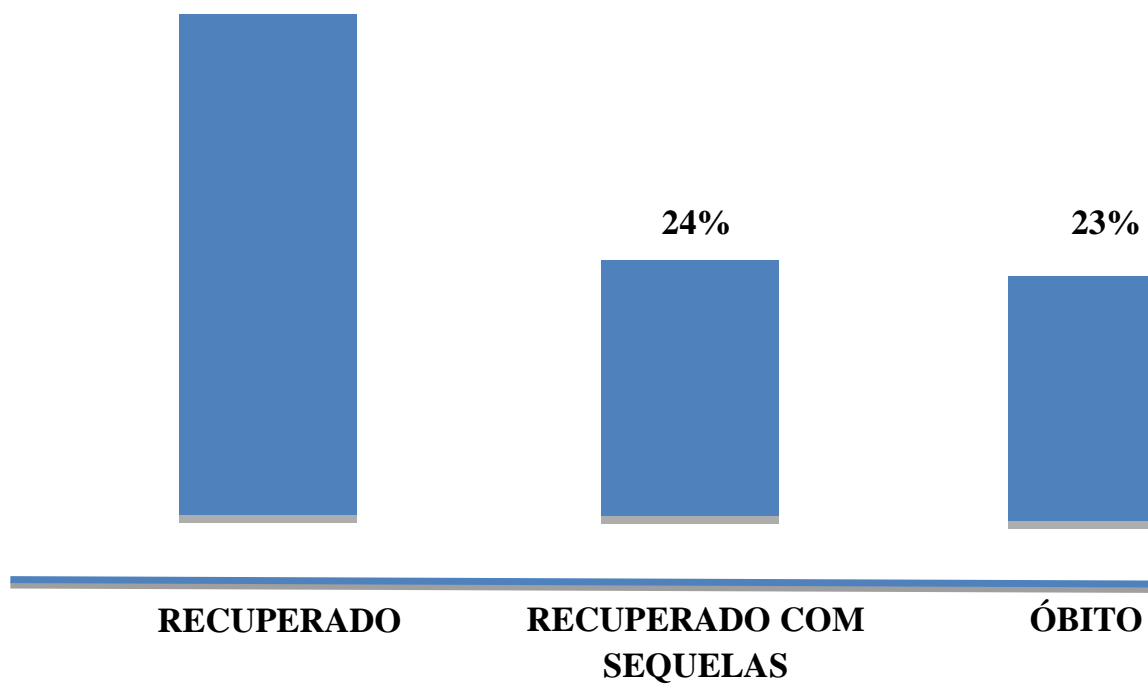

Fonte: Autores.

\section{Discussão}

Os inibidores de checkpoint, anticorpos monoclonais voltados a correceptores inibitórios envolvidos na modulação do sistema imune, melhoraram substancialmente o prognóstico para pacientes com câncer avançado. Acredita-se que o desenvolvimento das reações adversas imunomediadas seja provocado pelo aumento da atividade do sistema imunológico contra as células tumorais. Fatores como autoimunidade e a reação inflamatória contra o tecido do hospedeiro mediada por células T, anticorpos e citocinas são responsáveis pelo desencadeamento dessas toxicidades (Wainstein, et al. 2017).

Em relação aos pacientes do estudo, foi evidenciado que grande parte da população se encontrava na faixa etária de 60-89 anos, o que coincide com a idade de maior incidência dos tumores em tratamento. Em relação às comorbidades mais prevalentes, DM, HAS e TEV, essas não se correlacionaram com o uso de imunoterápicos, mas possuem em comum alguns fatores de risco para desenvolvimento dos tipos de cânceres mais frequentes em tratamento.

O percentual de pacientes que desenvolveram reações adversas imunomediadas, bem como as medidas terapêticas adotadas coincidem com o que Shoushtari, et al. (2018) observou em um estudo envolvendo 64 pacientes que foram tratados com nivolumabe e ipilimumabe para melanoma, $91 \%$ dos pacientes tiveram reações clinicamente significativas e quase $72 \%$ dos pacientes necessitaram de pelo menos uma dose de corticosteróides sistêmicos por um período de três meses. Cerca de um terço dos pacientes nesse estudo necessitaram da suspensão temporária ou permanente da terapia.

No presente estudo percebeu-se uma maior frequência nas reações dermatológicas, reações gastrintestinais, reações endócrinas e metabólicas e reações pulmonares, o que condiz com o estudo de Xu, et al. (2018) que compararam os inibidores de checkpoints com a terapia convencional e observou que os eventos adversos relacionados ao tratamento para drogas ICI ocorreram principalmente nos sistemas cutâneo, endócrino, hepático e pulmonar. 
Ao analisar os subgrupos das RAim relacionadas ao tratamento específico, os dados diferiram dos dados encontrados no Xu, et al. (2018) em que o atezolizumabe apresentou o maior risco de causar hipotireoidismo, náuseas e vômitos. Os principais eventos adversos causados por pembrolizumabe foram artralgia, pneumonite e toxicidade hepática. Nivolumabe e ipilimumabe tiveram resultados de toxicidade não sobrepostos. O nivolumabe causou principalmente toxicidades endócrinas. $\mathrm{O}$ ipilimumabe causou principalmente toxicidade cutânea, gastrointestinal e renal.

A maioria das reações imunomediadas foram classificadas como moderadas (53\%) e (47\%) como reações graves. As altas taxas de incidência e a gravidade, segundo Ellithi, et al. (2020) sofrem a influência dos fatores relacionados ao individuo, aos inibidores de checkpoints utilizados, a sua dose e mecanismo de ação, a combinação de mais de um agente ICI e as doenças autoimunes.

Conforme Wainstein, et al. (2017) a imunotoxicidade é habitualmente classificada em graus de acordo com o Critério de Terminologia Comum para Eventos Adversos (CTCAE) e a abordagem inicial deve ser ajustada à gravidade da manifestação. Para toxicidades de grau 1, a severidade é baixa e medidas de suporte são suficientes; para grau 2, a severidade é moderada e devem ser administrada corticoterapia sistêmica; para grau 3 e 4, a imunotoxicidade é severa e devem ser administrados corticoterapia sistêmica ou imunossupressor adicional e deve haver suspensão dos ICIs (Wang, et al. 2018).

Em relação ao desfecho clínico, viu-se que a maioria dos pacientes com $53 \%$ se recuperaram das RAim e $23 \%$ dos pacientes foram a óbito. O número de eventos fatais relatados as RAim fatais permanece muito baixo para pacientes com câncer avançado. Contudo, a analise que Wang, et al. (2018) fizeram demonstra que a colite (incluindo colite com perfuração intestinal) foi a causa mais frequente de morte com monoterapia usando anti-CTLA-4 (23 de 58 mortes relacionadas com antiCTLA-4). Eventos cardíacos, incluindo miocardite e morte súbita ocorreram em 9 (16\%) pacientes, insuficiência hepática em 5 (9\%) e pneumonite em 3 (5\%). Das 45 mortes associadas a anti-PD-1 / PD-L1, 19 resultaram de pneumonite, 7 de eventos cardíacos e 2 de colite / diarreia. Das 19 mortes associadas à terapia combinada, 4 resultaram de pneumonite, 4 de eventos cardíacos, 2 de eventos hematológicos (anemia aplástica) e 3 de eventos neurológicos (Champiat, et al. 2016; Eigentler, et al. 2016).

Nesse contexto, é essencial que os pacientes e cuidadores familiares recebam educação oportuna e atualizada sobre imunoterapias, seu mecanismo de ação e o perfil clínico de possíveis reações adversas imunomediadas. Esta deve ocorrer antes do início da terapia e continuar durante o tratamento e sobrevivência. Os pacientes devem ser orientados a sinalizar a todos os profissionais de saúde que estão recebendo ou receberam um agente imunoterápico e a relatar quaisquer alterações no estado de saúde a cada um, favorecendo assim uma detecção precoce e cuidado direcionado para as possíveis RAim (Wang, et al. 2020).

\section{Conclusão}

De modo geral, as RAim consideradas como leves podem ser manejadas pela suspensão temporária ou redução da dose, e a terapia pode ser reiniciada assim que as reações forem controladas ou debeladas. Reações moderadas e graves necessitam da administração de tratamento com corticosteroides sistêmicos e/ou outro regime imunossupressor, além da suspensão permanente da imunoterapia.

Assim, visto que os ICIs passam a ser cada vez mais indicados no tratamento oncológico e tendo em vista que os mecanismos de toxicidade não estão plenamente elucidados, é vital que a equipe de saúde se mantenha atualizada em relação as manifestações clínicas e manejo terapêutico das RAim. Uma monitorização adequada, diagnóstico precoce e gestão clínica são vitais, principalmente em situações de alto risco e por vezes fatais. O diagnóstico precoce reduz os sintomas, o tempo de hospitalização e a duração do tratamento com esteroides. A introdução de terapêutica dirigida, acompanhamento farmacoterapêutico adequado e o monitoramento pela farmacovigilância favorecerão a identificação de reações adversas 
imunomediadas, e o uso seguro e adequado dos inibidores de checkpoints imunológicos.

São necessários mais novos trabalhos para ampliar o entendimento de quais pacientes são mais suscetíveis ao desenvolvimento de reações adversas imunomediadas associadas a terapias com inibidores de checkpoint utilizadas no tratamento contra o câncer. Acredita-se que surgirão mais inibidores de checkpoints imunológicos aprovados, alguns com espectro de atividade mais alargado e com mecanismos de ação mais complexos, bem como continuará a busca por outros alvos terapêuticos dos checkpoints imunitários, o que demandará de outros estudos e acompanhamento da farmacovigilância.

\section{Referências}

Abdel, R. O. (2019). Toxicity patterns associated with chemotherapy/immune checkpoint inhibitor combinations: a meta-analysis. Immunotherapy, 11(6), 543554. 10.2217/imt-2018-0186

Barclay, J., Creswell, J., \& León, J. (2018). Cancer immunotherapy and the PD-1/PD-L1 checkpoint pathway. Archivos espanoles de urologia, 71(4), 393-399.

Brahmer, J. R., et al. (2018). Management of immune-related adverse events in patients treated with immune checkpoint inhibitor therapy: American Society of Clinical Oncology Clinical Practice Guideline. Journal of clinical oncology: official journal of the American Society of Clinical Oncology, 36(17), 1714. 10.1200/JCO.2017.77.6385.

Champiat, S. et al. (2016). Management of immune checkpoint blockade dysimmune toxicities: a collaborative position paper. Annals of Oncology, 27(4), 559-574. 10.1093/annonc/mdv623.

Dine, J., Gordon, R., Shames, Y., Kasler, M. K., \& Barton-Burke, M. (2017). Immune checkpoint inhibitors: an innovation in immunotherapy for the treatment and management of patients with cancer. Asia-Pacific journal of oncology nursing, 4(2), 127. 10.4103/apjon.apjon_4_17.

Eigentler, T. K. et al. (2016). Diagnosis, monitoring and management of immune-related adverse drug reactions of anti-PD-1 antibody therapy. Cancer treatment reviews, 45, 7-18. 10.1016/j.ctrv.2016.02.003.

Ellithi, M. et al. (2020). Toxicities of Immune Checkpoint Inhibitors: Itis-Ending Adverse Reactions and More. Cureus, 12(2). 10.7759/cureus.6935.

Hargadon, K. M., Johnson, C. E., \& Williams, C. J. (2018). Immune checkpoint blockade therapy for cancer: an overview of FDA-approved immune checkpoint inhibitors. International immunopharmacology, 62, 29-39. 10.1016/j.intimp.2018.06.001

Lleo, A., Rimassa, L., \& Colombo, M. (2019). Hepatotoxicity of immune check point inhibitors: Approach and management. Digestive and Liver Disease, 51(8), 1074-1078. https://doi.org/10.1016/j.dld.2019.06.017

Pereira A. S., Shitsuka, D. M., Parreira, F. J., \& Shitsuka, R. (2018). Metodologia da pesquisa científica. UFSM.

Regalla, D. K. R., Williams, G. R., \& kumar P. R. (2018). Immune checkpoint inhibitors in the management of malignancies in transplant recipients. Postgraduate Medical Journal, 94(1118), 704-708. 10.1136/postgradmedj-2018-136081

Shoushtari, A. N. et al. (2018). Measuring toxic effects and time to treatment failure for nivolumab plus ipilimumab in melanoma. JAMA oncology, 4(1), 98101. 10.1001/jamaoncol.2017.2391

Teixeira, H. C., Silva D. L., Menão, T. L., \& Oliveira, E. E. (2019). Proteínas de checkpoint imunológico como novo alvo da imunoterapia contra o câncer: revisão da literatura. HU Revista, 45(3), 325-333. https://doi.org/10.34019/1982-8047.2019.v45.28820.

Wang, D. Y. et al. (2018). Fatal toxic effects associated with immune checkpoint inhibitors: a systematic review and meta-analysis. JAMA oncology, 4(12), 1721-1728. 10.1001/jamaoncol.2018.3923.

Wang, G. et al. (2020). The expression and immunoregulation of immune checkpoint molecule VISTA in autoimmune diseases and cancers. Cytokine \& growth factor reviews, 52, 1-14. 10.1016/j.cytogfr.2020.02.002.

Wainstein, A. J. et al. (2017). Brazilian guidelines for the management of immune-related adverse events associated with checkpoint inhibitors. Brazilian Journal of Oncology, 13(43), 1-15.

Xu, C. et al. (2018). Comparative safety of immune checkpoint inhibitors in cancer: systematic review and network meta-analysis. Bmj, 363 . https://doi.org/10.1136/bmj.k4226. 